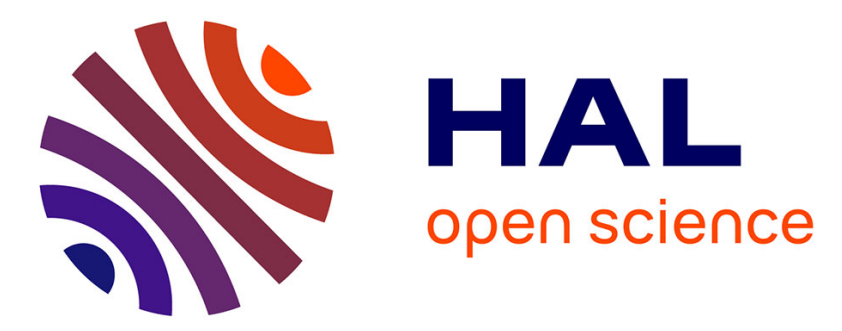

\title{
Energy-aware Service Level Agreements in 5G NFV architecture
}

\author{
Yacine Anser, Jean-Luc Grimault, Samia Bouzefrane, Chrystel Gaber
}

\section{To cite this version:}

Yacine Anser, Jean-Luc Grimault, Samia Bouzefrane, Chrystel Gaber. Energy-aware Service Level Agreements in 5G NFV architecture. 8th International Conference on Future Internet of Things and Cloud (FiCloud), Aug 2021, Rome, Italy. pp.377-382, 10.1109/FiCloud49777.2021.00061 . hal03381636

\section{HAL Id: hal-03381636 https://hal.science/hal-03381636}

Submitted on 17 Oct 2021

HAL is a multi-disciplinary open access archive for the deposit and dissemination of scientific research documents, whether they are published or not. The documents may come from teaching and research institutions in France or abroad, or from public or private research centers.
L'archive ouverte pluridisciplinaire HAL, est destinée au dépôt et à la diffusion de documents scientifiques de niveau recherche, publiés ou non, émanant des établissements d'enseignement et de recherche français ou étrangers, des laboratoires publics ou privés. 


\section{Energy-aware Service Level Agreements in 5G NFV architecture}

\author{
Yacine Anser \\ CEDRIC Lab and R\&D Orange Labs \\ Orange \& Cnam \\ Caen, France \\ yacine.anser@orange.com
}

\author{
Jean-Luc Grimault \\ $R \& D$ Orange Labs \\ Orange \\ Caen, France
}

jeanluc.grimault@orange.com samia.bouzefrane@cnam.fr chrystel.gaber@orange.com

\author{
Samia Bouzefrane \\ CEDRIC Lab \\ Cnam
}

Paris, France
Chrystel Gaber

$R \& D$ Orange Labs

Orange

Caen, France

yacine.s.

\begin{abstract}
With the emergence of new network paradigms, network operators are able to provide slices (multiple simultaneous logical networks) thanks to Network Function Virtualization (NFV) and Software-defined networking (SDN) technologies. Currently, network operators do not have the means to commit on energy consumption, although it is a growing request from their customers. Indeed, given that energy consumption does not appear in slice Service Level Agreements (SLA) nor in Virtual Network Function Descriptors (VNFD), slice automated management systems cannot take this criterion into account.

This paper addresses this issue with two contributions. First, we extend VNFD to include energy consumption. Second, we propose an SLA template for slices which includes energy based commitments. This template is complemented by some metrics in order to detect any deviation. Both contributions are illustrated by a use case. Our aim is to allow the entity in charge of referencing and placement of the virtual network functions (VNFs) for network service-composition purpose, to take into account energy consumption in addition to other preexisting aspects. This will help the network provider to commit on energy consumption with his customers through enhanced SLAs.

Index Terms-Energy-aware SLA, VNF, VNF Descriptor, SLA, NFV architecture.
\end{abstract}

\section{INTRODUCTION}

Network slicing is a technology that enables offering virtualized and independent logical networks on the same physical network infrastructure. The resources pertaining to the slice can be relevant to the different parts of the network. Indeed, the softwarization mechanisms in mobile edge networks, transport and core networks with technologies such as SDN or NFV enable the creation, the dynamic life-cycle management (e.g., deploying, changing, deleting) and the resources optimization (auto-scaling/migration/auto-healing) of network slices [1].

The NFV architecture was first explored by the European Telecommunication Standard Institute (ETSI) [2] with the following concept: any type of functional entity within Telco's network (e.g. gateway, firewall, $\mathrm{CDN}$, etc.) can be deployed on a virtual machine thanks, in particular, to the VNFD [3].

The slice is created based on an SLA that is negotiated between the slice provider and the slice owner. The slice owner specifies the requirements in terms of functionality, performance and security. For this purpose, the network slicing has particularly demanding requirements for real-time monitoring to meet customers' SLA. The Management and Orchestration
(MANO) entity manages the logical mapping between VNF instances assigned to tenants and the underlying virtual resources allocations, in compliance with the established SLA.

In addition to the already present considerations (cost, performance, reliability and security), the customer is more and more concerned about the impact of his activities on the environment. Therefore, he seeks to have services that consume as little energy as possible and with a low Carbon Footprint (especially if parts of the resources building the slice are located in his premises). To plan the energy consumption and to demonstrate that he operates the slice in an energyefficient way, the slice operator needs that the SLAs negotiated with his customer include the energy consumption target and that the VNFs referenced in his catalog include a description of their energy consumption, parameter which does not currently exist. Presently, the slice provider does not have the possibility to display a commitment on energy consumption because he does not have access to this information.

Two contributions are presented in this paper. First, we extend the VNFD with energy consumption data to help the network operator building an energy-aware network service. Second, we propose an energy-aware SLA template so that the operator can commit on energy notion, completed with metrics to detect deviations from engagement. To our knowledge, existing works [4] [5] [6] focus on VNF placement optimization algorithms with the objective to seek the smallest possible energy consumption of a service network composed of VNFs, while our study proposes a solution to expose the energy consumption of the VNFs so that a slice provider can offer a service in which the energy consumption characteristics are specified in the SLA. Other existing works are related to the cloud and hence need to be adapted to target the slice management. This paper is organized as follows : section II discusses the related work. Section III describes the NFV architecture and highlights the information provided by the VNFD. Section IV deals with our contribution. Section V illustrates the proposed model using a real use case. Finally, Section VI draws the conclusion. This research benefited from the innovations done within the H2020-INSPIRE-5Gplus research project [7]. 


\section{RELATED WORKS}

In this section, we give a short review of SLA schemes and briefly explore the state of the art including SLA for communication network and energy aware SLA for cloud environment.

\section{A. Service Level Agreement for communication networks}

SLAs are discussed in various technical domains such as web services [8], IoT [9] and cloud computing [10]. This section aims to describe the concept of SLA in the context of communication networks. Many definitions of SLA can be found in the scientific literature. ETSI defined the Service Level Agreement as a contract that describes the terms and conditions under which services are offered. This contract is created after a negotiation phase between providers and consumers [11]. The consumer firstly chooses an SLA level and expresses his requirement through Service level Objective (SLO) based on his strategies and needs (QoS requirements, price willing to pay, etc.). The provider is generally an operator and the consumer can be an ISP (Internet Service Provider), a company or simply an end user (subscriber). The SLO represents the conditions of end-to-end QoS (Quality of Service) requested by the user [11].

Authors of [12] did an overview of SLA in 5G. They identified few research papers that deal with this topic [13]-[19]. In [13], authors propose an end-to-end structure of SLA for slice-based $5 \mathrm{G}$ network with the objective of equality between the provider and the consumer. In order to detect agreement breaches, the authors propose metrics that they believe to be critical during the agreement such as throughput, penalty, cost, revenue, profit and QoS. The authors, in [19], highlight the fact that the SLA for network communication should be adjusted by exposing the properties of the future $5 \mathrm{G}$ network such as the capability to support complex "service provisioning" models including a mix of infrastructure resources, network services and even applications. Therefore, they proposed SLA parameters for $5 \mathrm{G}$ backhaul/fronthaul services, 5G transport network and cloud services. These parameters include the following: time period, periodicity, location, availability, reliability, cloud service resources, scaling rules and operational rules. With these parameters, a new SLA template for 5G network service has been proposed. In [20], authors did a survey on the existing SLAs for the network domain. They found from different network service providers the following performance indicators in their SLAs : Network Availability, Delay, Latency, Packet Delivery Ratio, Jitter, Congestion, Flow Completion time, Response time, Bandwidth, Utilization, MTBF (Mean Time Between Failure), MTRS (Mean Time to Restore Services), Solution time, Resolution time, LAN/WAN period of operation, LAN/WAN Service Time, Internet access across Firewall, RAS (Remote access Services).

Based on our literature review, we conclude that there is no work that proposes an SLA template as well as metrics to handle the energy dimension in network communications.

\section{B. Energy-aware Service Level Agreement for cloud environ-} ment

Even if a great number of works have been carried out to consider energy in cloud computing, a few works are close to our study as discussed below. In [21], authors define the energy-aware Service Level Agreement as an SLA that reflects the agreed scope for the cloud provider to operate in energy aware way and at the same time, guarantee a certain level of QoS for the IT customer. With the energy-aware SLA, they propose an approach which consists in relaxing certain jobs in a standardized way to obtain high energy consumption reduction without disturbing the efficiency and the availability of the system especially during the peak load times. A focus on energy reduction at Service Level Agreement is made by Sudhir Goyal et al. in [22]. They propose a Green Service Level Agreement (GSLA) template and negotiation strategies in order to offer resource services in an energy efficient manner to cloud users. They also implemented a framework as a proof of concept for academic environment and develop a GSLAaware Cloud Resource Reservation algorithm to provide resources energy efficiently. For monitoring the eco-efficiency of a data center service, Laszewski and Wang propose the concept of the GreenIT-SLA [23]. They develop green IT services and use GreenIT-SLAs for monitoring the service. This is done by including Green IT metrics as part of SLA in order to detect any breach of commitments. In [24], Christian Bunse et al. propose a proof of concept that shows that the SLA can be used to control energy optimization activities of a service provider by developing an energy-aware "GreenSLAs". For the authors, the GreenSLAs is a type of SLA between a service provider and his customers, that offers an extended scope of energy optimization to the service provider by relaxing traditional performance parameters. They integrate the metrics and behavior into text-based SLA specifications in the form of XML semantic information and present how to collect energydata on a per service request basis. A survey of existing Green SLA in the IT industry is also made in [20]. The authors confirm that the existing research works on green SLA are mainly based on cloud computing environment. Their work shows that most of the performance indicators in existing GSLAs are mainly focused on energy/power, carbon footprint, green energy, recycling issues.

\section{VNF SERVICE MANAGEMENT}

NFV architecture changes the traditional network infrastructure by replacing physical equipment with network programs hosted on VMs (Virtual Machine). A VNF is the software implementation of a network function. It is composed of one or multiple components called VNFC which run over the NFV Infrastructure (NFVI). The MANO covers the orchestration and the life-cycle management of physical and/or software resources and the life-cycle of VNFs. Figure 1 depicts the architecture as defined by ETSI [2].

To deploy a VNF on his infrastructure, the operator gets from the VNF provider a VNF package. A VNF Package is an archive that contains all the files and descriptors that are 


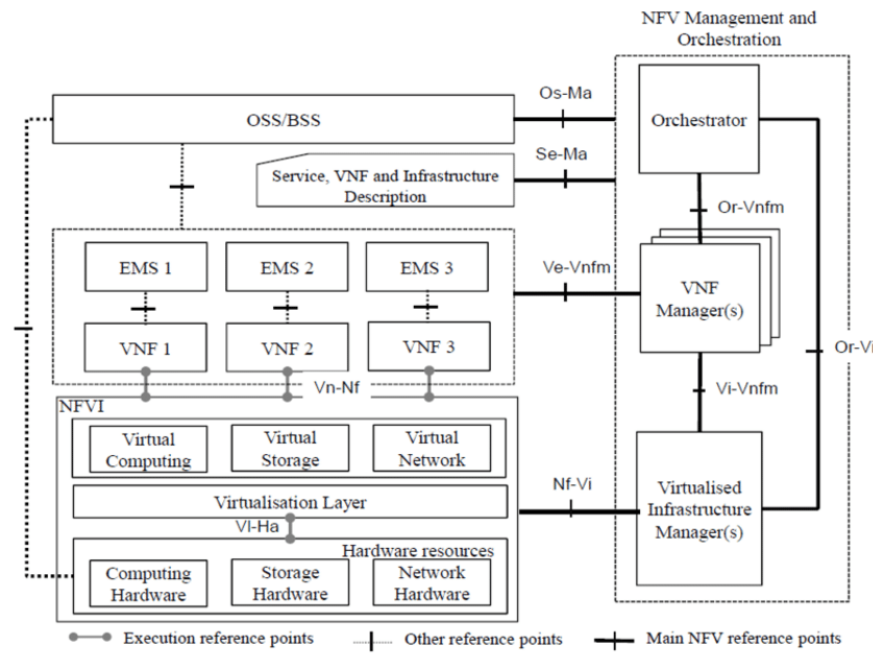

Fig. 1. ETSI NFV architecture framework

necessary to validate, instantiate and manage the life cycle of a VNF. The main file in the VNF package is the VNFD. Defined in [3], the VNFD is a deployment model that describes a VNF in terms of deployment requirements (resources, connectivity and interfaces) and operational behavior. It also contains information about the life cycle management of the VNF (scaling, upgrading, etc.). Each VNF has exactly one associated VNFD. Table I gives an overview of the general information contained in the VNFD.

\begin{tabular}{|l|l|l|}
\hline Groups & Sub-groups & Attribute(s) \\
\hline VNF profil & Unique VNFD identifier & vnfdId \\
\hline & Description of VNF & $\begin{array}{l}\text { vnfProductInfoDescription, } \\
\text { vnfProductInfoName, } \\
\text { vnfProductName }\end{array}$ \\
\hline & $\begin{array}{l}\text { Description of VNF } \\
\text { provider }\end{array}$ & vnfProvider \\
\hline & Version & $\begin{array}{l}\text { vnfSoftwareVersion, vnfd- } \\
\text { Version }\end{array}$ \\
\hline VNF data & Deployment flavours & deploymentFlavour \\
\hline & Deployment constraints & $\begin{array}{l}\text { vnfmInfo, } \\
\text { alLinkDesc }\end{array}$ \\
\hline & Resources needed & $\begin{array}{l}\text { virtualComputeDesc, vir- } \\
\text { tualStorageDesc, }\end{array}$ \\
\hline & Life cycle of the VNF & $\begin{array}{l}\text { lifeCycleManagementScript, } \\
\text { lcmOperationCoordina- } \\
\text { tion }\end{array}$ \\
\hline & External interface & vnfExtCpd \\
\hline VNFC data & Unique VNFC identifier & securityGroupRule \\
\hline & vdu.vduId \\
\hline & Rescription of VNFC & vdu.name, vdu.description \\
\hline & Internal interface & $\begin{array}{l}\text { vdu.virtualComputeDesc, } \\
\text { vdu.virtualStorageDesc }\end{array}$ \\
\hline & vdu.intCpd \\
\hline & VNFD's GENERAL INFORMATION \\
\hline
\end{tabular}

As shown here, the VNFD deployment model as standardized by ETSI lacks in terms of energy handling. It is consequently necessary to extend the VNFD so that it can take into account features related to energy.

\section{ENERGY-AWARE SLA AND VNFD EXTENSIONS}

This section details our two-fold contribution, that is, the VNFD energy extension and the energy-aware SLA.

\section{A. VNFD energy extension}

The VNFD energy extension that we propose is based on the ETSI ES 203539 document [25]. This document defines the energy metrics and measurement methods for NFV components including VNFs. It does not try to cover all different types of VNFs (e.g. firewall, gateway, etc.), but it provides the basis to make extensible definition.

The document introduces two metrics types : the energy efficiency metrics which are defined as functional units of useful output divided by the energy consumption and the resource efficiency metrics as the ratio of the useful outputs to the resources consumed by the VNF.

The measurement methods described in the document are related to power consumption and resource consumption. Before presenting a general measurement procedure, the document specifies measurement conditions by introducing recommendation on configuration of the System Under Test (SUT), the environment conditions of the test and the measurement instruments. More details can be found in the specification.

The following formulas, as presented in the ETSI document, translate more formally what has been mentioned above :

- The VNF's energy efficiency ratio metric is defined as :

$$
V N F_{-} E E R=\frac{\text { Usefuloutput }}{\text { Powerconsumption }}
$$

- The power consumption $P$ is measured as follows :

$$
P=P_{\text {load }}-P_{\text {idle }}
$$

Where $P_{\text {load }}$ is the power consumption of NFVI platform including the deployed VNF, and $P_{\text {idle }}$ is the power consumption of NFVI platform without any VNF deployment.

- The VNF's resource efficiency ratio metric is defined as:

$$
V N F \_R E R=\frac{\text { Usefuloutput }}{\text { Resourceconsumption }}
$$

- The resource consumption $R$ is measured as follows :

$$
R=R_{\text {load }}-R_{\text {idle }}
$$

Where $R_{\text {load }}$ is the resource consumption of NFVI platform including the deployed VNF, and $R_{\text {idle }}$ is the resource consumption of NFVI platform without any VNF deployment.

In addition, we propose the following metric in order to compute energy consumption :

$$
\mathrm{E}=\mathrm{P} \times \mathrm{T}
$$

Where $P$ is the power consumption and $T$ is the time.

The energy consumption is measured in Joule or KWh and the consumed resources refer to the virtual resources allocated 
to the VNF, that is : CPU, memory, storage and network. The useful output of the VNF depends on the different types of VNFs, that can be throughput (e.g. bps, pps) for data plane VNF, or capacity (e.g. subscribers, sessions) for control plane VNF [25]. We take the metrics 1, 3 and 5 which are complementary in expressing energy consumption and introduce them as an extension of the VNFD through a proposed methodology described in figure 2. This extension of the VNFD dedicated to energy aspects allows the operator to obtain information about the consumption features of the VNF as displayed by the manufacturer. To increase confidence in this information, the operator may rely on consolidation mechanisms based on past experiences with this particular VNF (or other VNFs of the same type from the same manufacturer), tests performed on the VNF or reputation from third parties experiences and opinions.

Each VNF runs on an NFVI and is scaled to provide different levels of service capacity by scaling one or more of its VNFCs. This capacity level depends on the provider of the VNF. Hence, the measurement tests must be performed with reference to an NFVI and with a given capacity level.

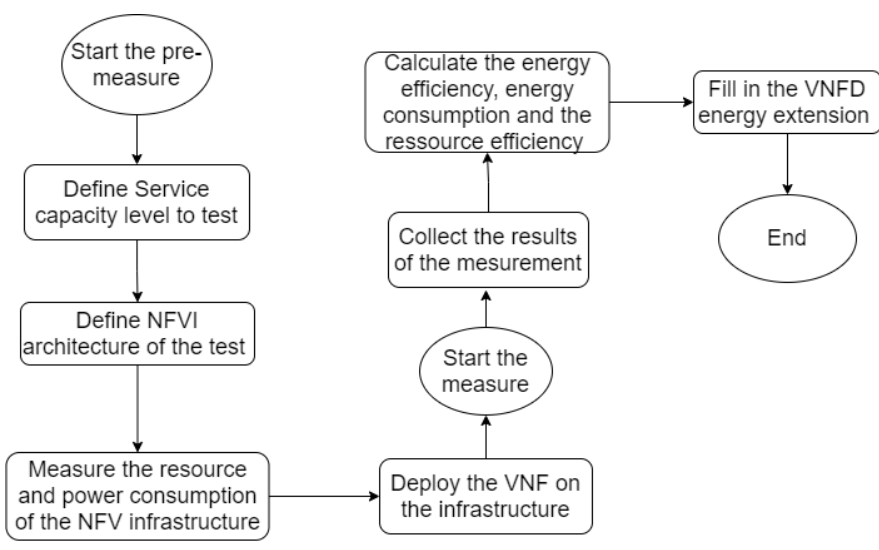

Fig. 2. Methodology for extending the VNFD

To extend the VNFD properly, we apply the conventions specified in [3]. The specification requires to provide for each attribute the following information :

- The attribute name;

- Indication whether the attribute is mandatory (M), optional $(\mathrm{O})$ or conditional $(\mathrm{C})$;

- The minimum and maximum cardinality of this information element (e.g. 1, 2, 0..N, 1..N). A cardinality range starting with 0 indicates that the attribute needs not always to be included;

- The type of the attribute values;

- A brief description of the attribute.

First, the manufacturer adds the NFVI reference. It's a description of the NFV infrastructure on which the measurement tests were made. The information of this attribute is provided by table II.

As mentioned before, all the measurements are made under a given capacity level. For that, the manufacturer has to specify

\begin{tabular}{|c|l|l|}
\hline Attribute & \multicolumn{2}{|c|}{ Description } \\
\hline \multirow{4}{*}{ NFVI reference } & Qualifier & $\mathrm{M}$ \\
\cline { 2 - 3 } & Cardinality & $1 \ldots \mathrm{N}$ \\
\cline { 2 - 3 } & Content & String \\
\cline { 2 - 3 } & Description & $\begin{array}{l}\text { Textual description of the NFV } \\
\text { infrastructure on which the } \\
\text { measurement tests were made }\end{array}$ \\
\hline
\end{tabular}

NFVI REFERENCE ATTRIBUTE

the capacity level employed during the measurement tests. Table III shows the structure of the capacity level attribute.

\begin{tabular}{|c|c|l|}
\hline Attribute & \multicolumn{2}{|c|}{ Description } \\
\hline \multirow{4}{*}{ Capacity level } & Qualifier & $\mathrm{M}$ \\
\cline { 2 - 3 } & Cardinality & $1 \ldots \mathrm{N}$ \\
\cline { 2 - 3 } & Content & String \\
\cline { 2 - 3 } & Description & $\begin{array}{l}\text { Textual description of the capacity level } \\
\text { of the VNF set up during the measure } \\
\text { TABLE III }\end{array}$ \\
\hline
\end{tabular}

The attributes that are related to energy such as Energy consumption, Energy efficiency, Resource efficiency and the duration of the measures are given in Table IV.

\begin{tabular}{|c|c|c|}
\hline Attributes & & Descriptions \\
\hline \multirow{4}{*}{ Time } & Qualifier & $\bar{M}$ \\
\hline & Cardinality & $1 \ldots \mathrm{N}$ \\
\hline & Content & Time \\
\hline & Description & $\begin{array}{l}\text { Duration of the measures ex- } \\
\text { pressed in hour }\end{array}$ \\
\hline \multirow{4}{*}{ Energy consumption } & Qualifier & $\bar{M}$ \\
\hline & Cardinality & $1 \ldots \mathrm{N}$ \\
\hline & Content & Float \\
\hline & Description & $\begin{array}{l}\text { The energy consumption (in Joule } \\
\text { or } \mathrm{kW} / \mathrm{h} \text { ) of the VNF recorded } \\
\text { after the measurements }\end{array}$ \\
\hline \multirow{4}{*}{ Energy efficiency } & Qualifier & M \\
\hline & Cardinality & $1 \ldots \mathrm{N}$ \\
\hline & Content & Float \\
\hline & Description & $\begin{array}{l}\text { The energy efficiency of the VNF } \\
\text { recorded after the measurements }\end{array}$ \\
\hline \multirow{4}{*}{ Resource efficiency } & Qualifier & $\mathrm{M}$ \\
\hline & Cardinality & $1 \ldots \mathrm{N}$ \\
\hline & Content & Float \\
\hline & Description & $\begin{array}{l}\text { The resource efficiency of the VNF } \\
\text { recorded after the measurements }\end{array}$ \\
\hline
\end{tabular}

THE ENERGY ATTRIBUTES

With these attributes added to the VNFD, we believe that the operator has enough information to refer the right VNF for its infrastructure.

\section{B. Energy-aware Service Level Agreement template for Net- work communication}

The template that we propose is an extended version of the network communication SLA. We combined both ideas found in the SLA generic model for ICT [11] and in the paper [22] where the authors propose a Green SLA for cloud computing.

The current model proposed by ETSI contains information about : the signatory parties that can include the provider and the end-user, an optional trusted third party that can include 
the monitoring provider, the services terms which consist of a composition of services matching the user demand (SLO). This demand has to be in relation with the end-to-end QoS of the service and with the penalties and costs in the case of a violation of the SLA. We propose to add two fields. The first field is the energy aware SLA ID which is a unique identifier to differentiate between contracts. The second field is the energy aware offer, which will make possible to express the customer's demands in relation to energy consumption. From that, the customer can accept, renegotiate or reject offers related to energy. The metrics we propose to detect any deviation from the commitment are related to the attributes added to the VNFD, they are listed in table V.

\begin{tabular}{|l|l|c|}
\hline Metric name & \multicolumn{1}{|c|}{ Description } & Unit \\
\hline $\begin{array}{l}\text { Total energy } \\
\text { consumption }\end{array}$ & $\begin{array}{l}\text { Total of energy consumed by } \\
\text { the VNFs provided by the op- } \\
\text { erator }\end{array}$ & Joule or KWH \\
\hline $\begin{array}{l}\text { Total energy } \\
\text { efficiency }\end{array}$ & $\begin{array}{l}\text { Total energy efficiency of VNF } \\
\text { provided by the operator }\end{array}$ & Mbps/Joule \\
\hline $\begin{array}{l}\text { Total resource } \\
\text { efficiency }\end{array}$ & $\begin{array}{l}\text { Total resource efficiency of } \\
\text { VNF provided by the operator }\end{array}$ & Mbps/MHz \\
\hline
\end{tabular}

ENERGY AWARE SLA METRICS

These parameters can be measured by the service provider (here the client must trust the operator on the results provided) or by the client himself. For this, he can rely on a subcontractor assessing the measurements.

\section{USE CASE}

In this section, we develop a realistic use case to illustrate the application of the energy aware SLA and the VNFD extension.

\section{A. General description}

We consider a use case where a hospital center wants to provide to its patients' rooms a remote monitoring service. The aim is to collect and store data in a private cloud for future analysis and processing. To deliver this end-toend service, the hospital owns a slice from an infrastructure operator (slice provider). The hospital needs to enrich this slice with components related to its activity, and to benefit from network components that will enable high debit and fluid communication. The network components of the slice are provided by the infrastructure operator and the related activity components by the hospital center. We assume that the operator uses only VNFs as network functions.

The hospital center is ecologically aware and practises a serious environmental policy. In addition, it needs to comply with its annual energy budget. Hence, it is looking for a commitment from the slice provider that includes the energy consumption of the components being supplied and hosted in his premises. Therefore, the operator must use the energy aware SLA proposed in section IV-B.

To be able to respect his commitment, the operator has to be aware of the energy consumption of the VNF that he purchased from a manufacturer. Thus, the manufacturer must add energy information into the VNFD following the methodology proposed in section IV-A. Figure 3 summarizes the use case.

For the service, the operator provides four network components for the hospital center : a security function with vFirewalls, a traffic analysis function with vDPI, an edge encryption function with vEncryption and a routing function with vGateways. These VNFs are provided by a manufacturer to the operator. The monitoring system is provided by the hospital center. Two capacity levels are identified : minimum where the number of VNFC is equal to one and maximum where the number of VNFC is equal to three.

The hospital center wants to supply 500 patients' rooms, with a throughput of $2 \mathrm{Mbit} / \mathrm{s}$ for each room. The hospital center needs a commitment on the energy consumption of the network components, so that their total energy consumption has to be less than 998,4 KWh/year; here the energy and the resource efficiency are not considered. After a negotiation phase between the operator and the hospital center, the following energy-aware SLA is created:

- The ID "1" is assigned to the SLA;

- The hospital center and the operator put their name down as signatory parties;

- The third party not included in this example (optional);

- The following service terms are added : the service is available $7 / 7$ and $24 / 24$ with a debit of $1 \mathrm{Gbit} / \mathrm{s}$, the total time to resolve a problem is less than one hour;

- The energy consumption as defined above is included;

- As penalty, the operator has to pay $10000 \$$ the hospital center for each failure to comply with the service terms.

Before the agreement between the operator and the hospital is concluded, the manufacturer has provided the VNF and therefore the VNFD to the operator. In order to add the energy consumption information into the VNFD, the manufacturer performed various measurement tests, the results are summarized in the table VI. The duration of the test is one hour. The capacity level corresponds to the throughput that each VNF can offer. The minimum capacity level is equal to $1 \mathrm{Gbit} / \mathrm{s}$ while the maximum is $2 \mathrm{Gbit} / \mathrm{s}$.

\begin{tabular}{|c|c|c|c|}
\hline VNFs & $\begin{array}{c}\text { Capacity } \\
\text { level }\end{array}$ & $\begin{array}{c}\text { Power consumption } \\
\text { (watts) }\end{array}$ & $\begin{array}{c}\text { Energy consumption } \\
\text { (kwh) }\end{array}$ \\
\hline \multirow{2}{*}{ vFirewall } & Min & 18 & 0.018 \\
\cline { 2 - 4 } & Max & 40 & 0.04 \\
\hline \multirow{2}{*}{ vDPI } & Min & 20 & 0.02 \\
\cline { 2 - 4 } & Max & 50 & 0.05 \\
\hline \multirow{2}{*}{ vEncryption } & Min & 39 & 0.039 \\
\cline { 2 - 4 } & Max & 90 & 0.09 \\
\hline \multirow{2}{*}{ vGateway } & Min & 9 & 0.009 \\
\cline { 2 - 4 } & Max & 20 & 0.020 \\
\hline
\end{tabular}

RESULTS OF MEASUREMENT TESTS

Several combinations of capacity levels of the VNFs can meet the commitments in terms of debit and energy. For example, selecting the minimum capacity level for all the VNFs can fit the needs. The following confirms this:

$24 \times 365 \times(0.0180+0.02+0.039+0.009)=753.36 k w H$ 


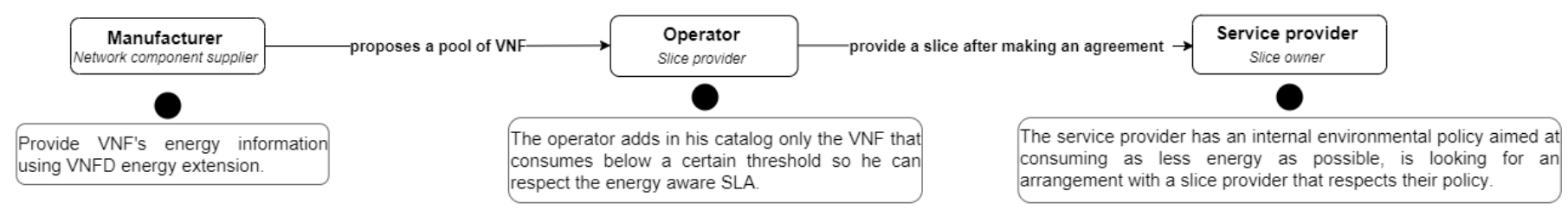

Fig. 3. Energy aware end-to-end service

This use case demonstrates that, with the help of the VNFD energy extension, the operator is able to reference the right VNF in order to fulfill the commitment. It's includes energy demand thanks to the energy aware SLA.

\section{CONCLUSION}

In this paper, a two-fold contribution has been made. First, we extended the VNFD in order to include energy information such as energy consumption, energy efficiency and resource efficiency. Second, we defined an energy aware SLA template so that the operator can commit on energy features. The objective is to enable the operator to reference the right VNF in terms of energy consumption in order to respect a commitment made with a consumer through energy aware SLA. The two key notions have been applied and illustrated through a realistic use case. As future works, we plan to improve MANO by handling the energy information present in the VNFD, and to extend the SLA language like WSagreement to formulate the energy aware SLA.

\section{REFERENCES}

[1] "5g network slicing using sdn and $\mathrm{nfv}$ : A survey of taxonomy, architectures and future challenges." [Online]. Available: https://www.sciencedirect.com/science/article/pii/S1389128619304773

[2] "Etsi gs nfv $002 \quad$ v1.2.1 : functions virtualisation $(\mathrm{nfv})$; architectural framework." [Online]. Available: https://www.etsi.org/deliver/etsi_gs /NFV/001_099/002/01.02.01_60/gs_NFV002v010201p.pdf

[3] "Etsi gs nfv-ifa 011 : Network functions virtualisation (nfv) management and orchestration vnf packaging specification."

[4] S. Kim, S. Park, Y. Kim, S. Kim, and K. Lee, "VNF-EQ: dynamic placement of virtual network functions for energy efficiency and QoS guarantee in NFV," Cluster Computing, vol. 20, no. 3, pp. 2107-2117, Sep. 2017.

[5] O. Soualah, M. Mechtri, C. Ghribi, and D. Zeghlache, Energy Efficient Algorithm for VNF Placement and Chaining, May 2017, pages: 588.

[6] M. M. Tajiki, S. Salsano, L. Chiaraviglio, M. Shojafar, and B. Akbari, "Joint Energy Efficient and QoS-aware Path Allocation and VNF Placement for Service Function Chaining." [Online]. Available: http://arxiv.org/abs/1710.02611

[7] J. Ortiz, R. Sanchez-Iborra, J. Bernal Bernabe, A. Skarmeta, C. Benzaid, T. Taleb, P. Alemany, R. Muñoz, R. Vilalta, C. Gaber, j.-p. Wary, D. Ayed, P. Bisson, M. Christopoulou, G. Xilouris, E. Montes de Oca, G. Gür, G. Santinelli, V. Lefebvre, and D. Lopez, INSPIRE-5Gplus: intelligent security and pervasive trust for $5 G$ and beyond networks, Aug. 2020.

[8] "The WSLA Framework: Specifying and Monitoring Service Level Agreements for Web Services." [Online]. Available: https://link.springer.com/article/10.1023/A:1022445108617

[9] G. Gaillard, D. Barthel, F. Theoleyre, and F. Valois, "SLA Specification for IoT Operation - The WSN-SLA Framework," report, INRIA, Jul. 2014, pages: 71. [Online]. Available: https://hal.inria.fr/hal-01024259

[10] "CSLA: A Language for Improving Cloud SLA Management." [Online]. Available: https://www.semanticscholar.org/paper/CSLA\%3AA-Language-for-Improving-Cloud-SLA-Management-KoukiLedoux/843eb54c6cda393db35015b61ea375b522557420?p2df
[11] "ETSI EG 202 009-3 : User group; quality of ict services part 3: Template for service level agreements (sla)." [Online]. Available: https://www.etsi.org/deliver/etsi_eg/202000_202099/20200903/01.03.00 50/eg_20200903v010300m.pdf

[12] H. N. Qureshi, M. Manalastas, S. M. A. Zaidi, A. Imran, and M. O. Al Kalaa, "Service Level Agreements for 5G and Beyond: Overview, Challenges and Enablers of 5G-Healthcare Systems," vol. 9. [Online]. Available: https://ieeexplore.ieee.org/document/9305243/

[13] M. A. Habibi, B. Han, M. Nasimi, and H. D. Schotten, "The Structure of Service Level Agreement of Slice-based 5G Network," arXiv:1806.10426 [cs], Jun. 2018, arXiv: 1806.10426

[14] M. Xie, Q. Zhang, A. J. Gonzalez, P. Grønsund, P. Palacharla, and T. Ikeuchi, "Service Assurance in 5G Networks: A Study of Joint Monitoring and Analytics," in 2019 IEEE 30th Annual International Symposium on Personal, Indoor and Mobile Radio Communications (PIMRC), Sep. 2019, pp. 1-7, iSSN: 2166-9589.

[15] E. Kapassa, M. Touloupou, A. Mavrogiorgou, and D. Kyriazis, "5G SLAs: Automated proposition and management of agreements towards QoS enforcement," in 201821 st Conference on Innovation in Clouds, Internet and Networks and Workshops (ICIN), Feb. 2018, pp. 1-5, iSSN: 2472-8144.

[16] E. Kapassa, M. Touloupou, and D. Kyriazis, "SLAs in 5G: A complete framework facilitating VNF- and NS- tailored SLAs management," p. 7.

[17] M. Touloupou, E. Kapassa, C. Symvoulidis, P. Stavrianos, and D. Kyriazis, "An Integrated SLA Management Framework in a 5G Environment," in 2019 22nd Conference on Innovation in Clouds, Internet and Networks and Workshops (ICIN), Feb. 2019, pp. 233-235, iSSN: 24728144.

[18] B. Khodapanah, A. Awada, I. Viering, D. Oehmann, M. Simsek, and G. P. Fettweis, "Fulfillment of Service Level Agreements via SliceAware Radio Resource Management in 5G Networks," in 2018 IEEE 87th Vehicular Technology Conference (VTC Spring), Jun. 2018, pp. 16, iSSN: 2577-2465.

[19] I. Mesogiti, E. Theodoropoulou, K. Filis, G. Lyberopoulos, R. C. Palancar, N. A. S. Linares, D. Camps-Mur, J. Gutirrez, and A. Tzanakaki, "Network Services SLAs over 5G Infrastructure Converging Disaggregated Network and Compute Resources," in 2018 IEEE 23rd International Workshop on Computer Aided Modeling and Design of Communication Links and Networks (CAMAD), Sep. 2018, pp. 1-5, iSSN: 2378-4873.

[20] I. Ahmed, H. Okumura, and K. Arai, "Analysis on Existing Basic Slas and Green Slas to Define New Sustainable Green SLA," International Journal of Advanced Computer Science and Applications, vol. 6, no. 12, p. 9, 2015.

[21] N. Joy, K. Chandrasekaran, and Binu A., "Energy Aware SLA with Classification of Jobs for Cloud Environment," Procedia Computer Science, vol. 70, pp. 740-747, 2015.

[22] S. Goyal, S. Bawa, and B. Singh, "Green Service Level Agreement (GSLA) framework for cloud computing," Computing, vol. 98, no. 9, pp. 949-963, Sep. 2016.

[23] G. von Laszewski and L. Wang, "GreenIT Service Level Agreements," in Grids and Service-Oriented Architectures for Service Level Agreements, Aug. 2010, pp. 77-88, journal Abbreviation: Grids and Service-Oriented Architectures for Service Level Agreements.

[24] C. Bunse, S. Klingert, and T. Schulze, "GreenSLAs: Supporting EnergyEfficiency through Contracts," in Energy Efficient Data Centers, ser. Lecture Notes in Computer Science, J. Huusko, H. de Meer, S. Klingert, and A. Somov, Eds. Berlin, Heidelberg: Springer, 2012, pp. 54-68.

[25] "Etsi es 203539 : Environmental engineering (ee); measurement method for energy efficiency of network functions virtualisation (nfv) in laboratory environment." 